\title{
Distribution and drug resistance of pathogenic bacteria in ventilator-associated pneumonia at a local hospital of North-eastern China
}

This article was published in the following Dove Press journal: Infection and Drug Resistance

\section{Yu Wang \\ Rong Zhang \\ Wei Liu}

Department of Emergency, Shengjing Hospital Affiliated to China Medical University, Heping District, Shenyang, Liaoning Province II 0004, People's Republic of China
Correspondence: Yu Wang Department of Emergency, Shengjing Hospital Affiliated to China Medical University, No. 36 Sanhao Street, Heping District, Shenyang, Liaoning Province I 1 0004, People's Republic of China Tel +8624966I5 642I6 Email wangy8@sj-hospital.org
Purpose: To study the distribution of pathogenic bacteria in ventilator-associated pneumonia (VAP), and epidemiological characteristics of extended-spectrum $\beta$ lactamase (ESBL)-producing bacteria.

Patients and methods: Lower respiratory tract secretions from 156 patients with mechanical ventilation were collected using a protective specimen brush (PSB), with quantitative bacterial culture carried out and antibiotic sensitivity measured. ESBLs produced by Gram-negative bacilli were detected using the double disk diffusion method and monitored by plasmid profiles. Results: Gram-negative bacilli accounted for $78.9 \%$ of VAP pathogens, with Acinetobacter baumannii (25\%), Pseudomonas aeruginosa (19.7\%), and Klebsiella pneumoniae (14.5\%) as the most common strains. There were 12 Gram-positive strains detected (15.8\%); mostly methicillinresistant. Staphylococcus aureus and methicillin-resistant coagulase-negative Staphylococcus. There were also four strains of Candida albicans detected (5.26\%). Most Gram-negative bacilli are sensitive to imipenem, but $A$. baumannii is serious resistant. ESBLs were detected in nine strains of Gram-negative bacilli; mainly produced by $K$. pneumoniae and Escherichia coli, to different degrees of multidrug resistance. Five strains of $K$. pneumoniae-producing ESBLs were from the same clonal origin, as confirmed by plasmid restriction endonuclease analysis. Conclusion: VAP was mainly caused by Gram-negative bacteria, with high antibiotic resistance rates. Plasmids played an important role in the spread of antibiotic resistance among bacteria. Keywords: ventilator-associated pneumonia, pathogenic bacteria, extended-spectrum $\beta$ lactamases, drug resistance plasmid

\section{Introduction}

Ventilator-associated pneumonia (VAP) is defined as pneumonia occurring more than 48 hours after the onset of mechanical ventilation. VAP is a common complication in patients with acute and chronic respiratory failure, mostly with refractory pathogens and high antibiotic resistance. ${ }^{1}$ The incidence and mortality of VAP are higher in the intensive care unit (ICU) because of immune deficiency, severe basic diseases, and increased use of artificial airway or mechanical ventilation, which are associated with longer hospital stays and increase health care costs significantly. Hence, knowledge of antibiotic resistance and the characteristics of drug resistance are important for VAP prophylaxis and treatment. Previous studies have found that bacterial plasmid or chromosome-mediated extended-spectrum $\beta$ lactamases (ESBLs) are the major cause of $\beta$ lactam antibiotic resistance. ${ }^{2}$ Drug selective pressure has resulted in the increasing separation rate of ESBL-producing bacteria from clinical specimens. Hospital 
infections caused by ESBL-producing bacteria worldwide are reported with increasing frequency, and the problem of drug resistance caused by ESBLs has attracted increasing attention. $^{3-5}$

The purpose of this study was to explore the distribution of pathogens associated with VAP, to determine the epidemiological characteristics of ESBL-producing bacteria in hospital, and to guide the rational clinical use of antibiotics, as well as to control effectively the spread of bacterial resistance.

\section{Materials and methods Study design}

This was a retrospective, observational study that was performed using electronic medical record data from patients who were hospitalized at the Emergency Department. The protocol was reviewed and approved by the ethical committee of Shengjing Hospital Affiliated to China Medical University. The requirement for informed consent was waived due to the retrospective design and the fact that all patient data were anonymized and deidentified prior to the analysis.

\section{Patients}

Patients above the age of 18 years admitted in the emergency intensive care unit (EICU), requiring intubation and mechanical ventilation for more than 48 hours, were considered eligible for inclusion. Exclusion criteria included intubation in other areas of hospital, tracheotomy, or suffering from pneumonia on admission or during first 48 hours of mechanical ventilation. Patients were recruited consecutively and followed until VAP diagnosis, death, or discharge from EICU.

\section{Definition of VAP}

VAP was considered in the presence of a new or progressive and persistent ( $\geq 48$ hours) radiographic infiltrate consolidation, cavitation, or pleural effusion, plus at least two of the following: 1) leucocytosis $>10,000$ white blood cells $/ \mathrm{mm}^{3}$ or leucopenia $\left(<4,000\right.$ white blood cells $\left.\left./ \mathrm{mm}^{3}\right) ; 2\right)$ body temperature $\geq 38^{\circ} \mathrm{C}$ or $<35^{\circ} \mathrm{C} 3$ ) purulent tracheal secretion or change in character of sputum. Diagnosis was further confirmed by protective specimen brush (PSB) cultures $\geq 10^{3} \mathrm{CFU} / \mathrm{mL}$ as a positive threshold.

\section{Collection and processing of specimens}

The samples for microbiological follow-up were collected by PSB. Specimens were inoculated on blood and MacConkey agar plates using the four district scribing method, with the colony growth condition monitored, and the results were identified using the French API system (BioMerieux-Vitek,
Hazelwood, MO, USA) after 20 hours culture at $35^{\circ} \mathrm{C}$. Bacterial strains used as quality control were Escherichia coli ATCC25922, Pseudomonas aeruginosa ATCC27853, and Staphylococcus aureus ATCC25923.

\section{Detection of ESBLs and drug sensitivity test}

Determination test of ESBLs was used by double disk diffusion method. Susceptibility to antimicrobial agents was tested by Kirby-Bauer disk diffusion method. The results were interpreted according to the recommended 2011 guidelines of the Clinical and Laboratory Standards Institute, and the strains that were moderately sensitive to drugs were considered drug-resistant in statistical analysis.

\section{Analysis of bacterial plasmid features}

Bacterial plasmids were extracted using a Promega Plasmid Extraction Kit (Promega Corporation, Fitchburg, WI, USA). The plasmids were digested with HindIII restriction endonuclease and subjected to electrophoresis on $0.8 \%$ agarose gel, using a HindIII restriction enzyme digested $\lambda$ DNA as the standard.

\section{Data analysis}

Statistical analysis was performed using SPSS version 17.0 software (SPSS Inc., Chicago, IL, USA). Quantitative results were expressed as the mean \pm standard error. Categorical variables were expressed as counts and percentages which were analyzed using the $\chi^{2} . P<0.05$ was considered statistically significant.

\section{Results}

\section{Distribution of pathogens}

One-hundred and fifty-six patients receiving mechanical ventilation in EICU were included in the study from January 2014 to July 2016 at a local hospital in Northeast China, a tertiary care comprehensive hospital with 5,000 beds. During the study 1,365 mechanical ventilation-days were accumulated, and 76 patients (48.7\%; 95\% CI: $33.8-49.8$ ) were diagnosed with VAP. The crude incidence of VAP was 47.6 episodes per 1,000 ventilator-days. The general data of the 76 patients (whose ages ranged from 18 to 92 years, with a mean age of $59.3 \pm 18.0$ years) are shown in Table 1. Seventysix strains were isolated from these patients. There were 60 strains of Gram-negative bacilli (78.9\%) isolated from PSB specimens, most commonly Acinetobacter baumannii (25\%), P. aeruginosa (19.7\%), and Klebsiella pneumoniae (14.5\%), followed by E. coli (7.86\%); 9 ESBL-producing strains were 
Table I General data of the 76 VAP patients

\begin{tabular}{|l|l|l|}
\hline Variables & $\begin{array}{l}\text { Number of } \\
\text { patients }\end{array}$ & Rate (\%) \\
\hline Gender & & \\
\hline Male & 46 & 60.5 \\
\hline Female & 30 & 39.5 \\
\hline Basic diseases & & \\
\hline $\begin{array}{l}\text { Chronic obstructive pulmonary } \\
\text { disease }\end{array}$ & 2 I & 27.6 \\
\hline Severe pneumonia & $1 \mathrm{I}$ & 14.5 \\
\hline Sepsis with ARDS & 9 & 11.8 \\
\hline Cerebrovascular accidents & 8 & 10.5 \\
\hline Acute pancreatitis & 7 & 9.2 \\
\hline Trauma & 6 & 7.9 \\
\hline Bronchial asthma & 5 & 6.6 \\
\hline Malignant tumor & 5 & 6.6 \\
\hline Drug poisoning & 4 & 5.3 \\
\hline
\end{tabular}

Abbreviations: ARDS, acute respiratory distress syndrome; VAP, ventilatorassociated pneumonia.

isolated with a detection rate of $52.9 \%$, which were mainly produced by $K$. pneumoniae and E. coli. There were also 12 Gram-positive strains detected (15.8\%); mainly methicillin-resistant $S$. aureus (MRSA) and methicillin-resistant coagulase-negative Staphylococcus (MRCNS). Four strains of Candida albicans (5.26\%) were also detected (Table 2).

\section{Drug resistance of pathogenic bacteria}

The resistance rates of main Gram-negative bacilli to commonly used antibiotics are listed in Table 3. A. baumannii had the highest resistance with $63.2 \%$ (95\% CI: 41.5-84.6) resistance against imipenem, compared with $33.3 \%$ (95\% CI: 11.8-61.6) for $P$. aeruginosa. K. pneumoniae was sensitive to imipenem. Gram-positive bacteria were mainly $M R S A$ and $M R C N S$, which were $100 \%$ sensitive to vancomycin and teicoplanin.

\section{Comparison of antibiotic resistance of ESBL-producing and -nonproducing strains}

The resistance rates of ESBL-producing strains toward third-generation cephalosporins, such as ceftazidime, cefoperazone, and cefotaxime, were significantly higher than in non-ESBL-producing strains, with resistance rates of 88.9\% (95\% CI: $51.8-99.7), 100 \%$ (95\% CI: 66.4-100), and $100 \%$ (95\% CI: $66.4-100)$, respectively $\left(\chi^{2}=7.137,7.969\right.$, 5.885, $P<0.05)$. The resistance rates of ESBL-producing strains toward ciprofloxacin was significantly higher than for non-ESBL-producing strains (77.8\% [95\% CI: 40-97.2], $12.5 \%$ [95\% CI: $0.3-52.7])\left(\chi^{2}=7.244, P<0.05\right)$. However, the resistance rates of ESBL-producing strains against cefoxitin sodium and piperacillin/tazobactam were relatively low,
Table 2 PSB culture positive results $\left(\geq 10^{3} \mathrm{CFU} / \mathrm{mL}\right)$

\begin{tabular}{|l|l|l|}
\hline $\begin{array}{l}\text { Low respiratory tract } \\
\text { pathogens }\end{array}$ & $\begin{array}{l}\text { Number of } \\
\text { strains }\end{array}$ & (\%) \\
\hline Gram-negative bacilli & & \\
\hline A. baumanni & 19 & 25.0 \\
\hline P. aeruginosa & 15 & 19.7 \\
\hline K. pneumoniae & II (7 ESBL-producing) & 14.5 \\
\hline E. coli & 6 (2 ESBL-producing) & 7.89 \\
\hline Enterobacter cloacae & 4 & 5.26 \\
\hline Pseudomonas maltophilia & 3 & 3.94 \\
\hline Others & 2 & 2.63 \\
\hline Gram-positive & & \\
\hline MRSA & 6 & 7.89 \\
\hline MRCNS & 4 & 5.26 \\
\hline Enterococcus faecalis & 2 & 2.63 \\
\hline Fungi & & \\
\hline C. albicans & 4 & 5.26 \\
\hline Total & 76 & 100 \\
\hline
\end{tabular}

Abbreviations: ESBL, extended-spectrum $\beta$ lactamase; MRCNS, methicillinresistant coagulase-negative Staphylococcus; MRSA, methicillin-resistant S. aureus; PSB, protective specimen brush.

$22.2 \%$ (95\% CI: $2.8-60)$ and $11.1 \%$ (95\% CI: $0.3-48.3)$ respectively. As listed in Table 4, no strains with resistance to imipenem were observed.

\section{Plasmid restriction endonuclease analysis}

The restriction endonuclease digestion results of seven strains of ESBL-producing K. pneumoniae showed that five strains had high homology, demonstrated by the same number and length of DNA fragments after digestion. This suggests that ESBL-producing K. pneumoniae in the EICU had the same clonal origin. The remaining two strains had different enzyme digestion profiles (Figure 1).

\section{Discussion}

VAP has a high incidence and mortality rate in both domestic and foreign hospitals, resulting in prolonged time in the ICU and increased duration of mechanical ventilation, as well as increased hospitalization cost. Reports showed that the incidence of VAP was $6 \%-52 \%$ or (1.6-52.7) cases $/ 1,000$ ventilator-days, with a mortality rate of $14 \%-50 \%$. About $6-8$ VAPs resulted in prolongation of mechanical ventilation by 5.4-14.5 days, ${ }^{8}$ ICU retention time by $6.1-17.6$ days, and hospitalization time by 11-12.5 days. ${ }^{6,9}$ In the EICU of our hospital, during the study 1,365 mechanical ventilation-days were accumulated, and 76 patients (48.7\%; 95\% CI: 33.8-49.8) were diagnosed with VAP. The crude incidence of VAP was 47.6 episodes per 1,000 ventilator-days. All-cause mortality and attributable mortality associated with VAP were $42.1 \%$ (32/76), and $18.4 \%$ (14/76), respectively. Therefore, how to prevent and cure VAP, 
Table 3 Antibiotic resistance of major Gram-negative bacilli

\begin{tabular}{|l|l|l|l|}
\hline Antibiotic & A. baumannii $(\mathbf{n}=\mathbf{1 9})$ & P. aeruginosa $(\mathbf{n}=\mathbf{I 5})$ & K. pneumoniae $(\mathbf{n}=\mathbf{I} \mathbf{I})$ \\
\hline Imipenem & 63.2 & 33.3 & 9.1 \\
\hline Ceftazidime & 47.4 & 26.7 & 45.4 \\
\hline Cefoperazone & 78.9 & 60.0 & 63.6 \\
\hline Cefotaxime & 94.7 & 80.0 & 72.7 \\
\hline Cefepime & 47.4 & 40.0 & 36.4 \\
\hline Piperacillin/tazobactam sodium & 57.9 & 33.3 & 18.2 \\
\hline Ticarcillin/clavulanic acid & 94.7 & 66.7 & 54.5 \\
\hline Cefoperazone/sulbactam & 15.8 & 20.0 & 36.3 \\
\hline Cefoxitin & 84.2 & 86.7 & 45.4 \\
\hline Ciprofloxacin & 63.2 & 40.0 & 27.3 \\
\hline Amikacin & 21.1 & 20.0 & 27.3 \\
\hline Aztreonam & 73.7 & 46.7 & \\
\hline
\end{tabular}

Table 4 Comparison of antibiotic resistance of ESBL-producing and -nonproducing strains

\begin{tabular}{|c|c|c|c|c|}
\hline \multirow[t]{2}{*}{ Antibiotics } & \multicolumn{2}{|c|}{ ESBL-producing strains $(n=9)$} & \multicolumn{2}{|c|}{ Non-ESBL-producing strains $(n=8)$} \\
\hline & $\mathbf{S}$ & I+R (\%) & $\mathbf{S}$ & I+R (\%) \\
\hline Ceftazidime & $\mathrm{I}$ & $8(88.9)$ & 6 & $2(25.0)$ \\
\hline Cefoperazone & 0 & $9(100)$ & 5 & $3(37.5)$ \\
\hline Cefotaxime & 0 & $9(100)$ & 4 & $4(50.0)$ \\
\hline Ciprofloxacin & 2 & $7(77.8)$ & 7 & I (12.5) \\
\hline Cefoxitin & 7 & $2(22.2)$ & 4 & $4(50.0)$ \\
\hline Cefepime & 2 & $7(77.8)$ & 7 & I (I2.5) \\
\hline Piperacillin/tazobactam sodium & 8 & $I(11.1)$ & 7 & $I(12.5)$ \\
\hline Ticarcillin/clavulanic acid & 0 & $9(100)$ & 4 & $4(50.0)$ \\
\hline Cefoperazone/sulbactam & 5 & $4(44.4)$ & 6 & $2(25.0)$ \\
\hline Imipenem & 9 & $0(0)$ & 7 & I (I2.5) \\
\hline
\end{tabular}

Abbreviations: ESBL, extended-spectrum $\beta$ lactamase; I, intermediate; $R$, resistant; $S$, sensitive.

$\begin{array}{llllllll}7 & 6 & 5 & 4 & 3 & 2 & 1 & M\end{array}$

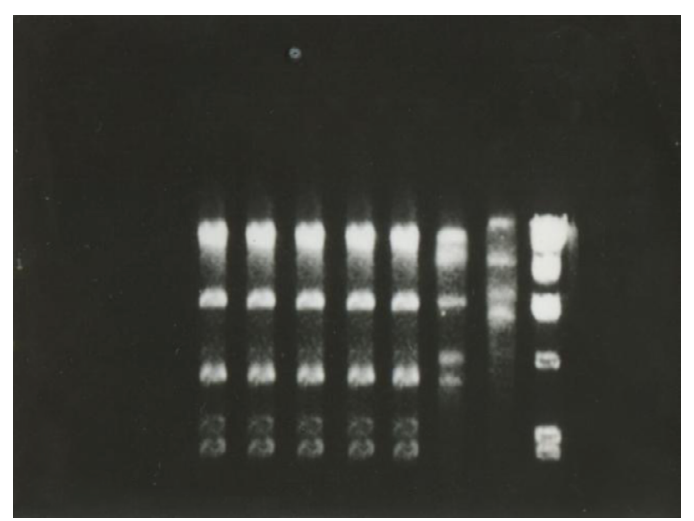

$23,130 \mathrm{bp}$

$9,416 \mathrm{bp}$

$6,557 \mathrm{bp}$

$4,361 \mathrm{bp}$

$2,322 \mathrm{bp}$

$2,027 \mathrm{bp}$

Figure I Plasmid restriction endonuclease fingerprinting of seven $K$. pneumoniae strains producing ESBLs.

Notes: Lanes I-7, K. pneumoniae strains producing ESBLs; M, $\lambda$ DNA/HindllI marker. Abbreviation: ESBL, extended-spectrum $\beta$ lactamase.

and further improve the successful rescue rate of critically ill patients, has become one of the hot topics in medical science.

The pathogens of VAP are mostly Gram-negative bacilli, and VAP may be caused by colonization in the lower respiratory tract. ${ }^{10}$ The current study used PSB to monitor the bacteria in the lower respiratory tract secretions of 156 patients with mechanical ventilation. The results showed that Gram-negative bacilli accounted for $78.9 \%$ of pathogenic bacteria, followed by Gram-positive cocci $(15.8 \%)$ and fungi $(5.26 \%)$. There were many types of Gram-negative bacilli detected, which were different from nonhospital pathogens, with A. baumannii (25\%), P. aeruginosa (19.7\%), and $K$. pneumoniae $(14.5 \%)$ as the most common ones. Chittawatanarat et $\mathrm{al}^{11}$ reported that the three most common VAP Gram-negative bacilli were A. baumannii, K. pneumoniae, and $P$. aeruginosa. Such results indicated different pathogenic bacteria and drug resistance patterns due to different prophylactic and empirical use of antibiotics in different areas.

A. baumannii is an opportunistic pathogen that is widely distributed in the hospital environment, on human skin, and in the respiratory tract, which can cause serious hospital infection in patients with low immunity. The current study showed that A. baumannii had high resistance to imipenem (63.2\%) and cefotaxime (94.7\%), while its resistance to cefoperazone/ 
sulbactam sodium was relatively low $(15.8 \%)$. The reason may be that sulbactam is more stable to most $\beta$ lactamase, and cannot be hydrolyzed by plasmid and chromosomal mediation of $\beta$ lactamase. The synergistic effect of Shubatan and cefoperazone was observed. Therefore, cefoperazone/ sulbactam sodium can be used as the first treatment option for carbapenem-resistant A. baumannii infection. A. baumannii demonstrates obvious resistant to most commonly used antibiotics. Its resistance mechanism is related to plasmidmediated production of TEM-1, TEM-2 type $\beta$ lactamase, chromosome-mediated production of AmpC enzymes, amino modified enzyme, penicillin-binding protein changes, and decreased permeability of the outer membrane proteins. ${ }^{12-14}$

$P$. aeruginosa is one of the major pathogenic bacteria in VAP. Our results showed that $P$. aeruginos $a$ accounted for $19.7 \%$ of Gram-negative bacilli, with a resistance rate of $<40.0 \%$ to imipenem, amikacin and ceftazidime, piperacillin/ tazobactam, and cefoperazone sodium/sulbactam. The mechanism of drug resistance of $P$. aeruginosa is complicated, mainly due to the fact that it is an alginate organism with adhesion coating produced by its cell membrane, allowing the bacteria to be easily attached to the respiratory mucosa, not easily swallowed, and forming a physical barrier. The formed physical barrier leads to the decreased permeability of antibiotics, and neutralization of some drugs, which also keeps the bacteria in the biofilm in an inactive growth phase, making them insensitive to antibiotics. ${ }^{15,16}$ The mechanism of drug resistance of $P$. aeruginosa involves many aspects; thus, it is better to use combination therapy, such as sensitive $\beta$ lactam or carbapenems plus aminoglycoside or quinolone drugs.

Our results showed that $K$. pneumoniae accounted for $14.5 \%$ and $E$. coli accounted for only $7.86 \%$ of pathogens detected, which ranked the third and fourth after P. aeruginosa. There were nine ESBL-producing strains, with a positive detection rate of $52.9 \%$. K. pneumoniae and E. coli were the main bacteria producing ESBLs. In this study, the resistance rates of ESBL-producing strains toward the third-generation cephalosporins, such as ceftazidime, cefoperazone, and cefotaxime, were significantly higher than for non-ESBL-producing strains, with resistance rates of $88.9 \%, 100 \%$, and $100 \%$, respectively $\left(\chi^{2}=7.137,7.969\right.$, $5.885, P<0.05)$. The resistant rate of ESBL-producing strains against cefepime was $77.8 \%$, which failed to confirm the hydrolytic stability of fourth-generation cephalosporins in the presence of ESBLs, ${ }^{17}$ while ESBL-producing bacteria showed some sensitivity toward cefoxitin and compound preparations containing $\beta$ lactamase inhibitors. Clavulanic acid has the disadvantage of inducing $\beta$ lactamase production in pathogenic bacteria, leading to emergence of resistance to clavulanic acid among ESBL-producing bacteria. Therefore, the antibacterial effects of compounds containing clavulanic acid are weaker than those of sulbactam or tazobactam. ${ }^{18}$ The results of the current study were consistent with such a theory. The resistance rates of ESBL-producing bacteria against sodium ticarcillin/clavulanic acid was as high as $100 \%$, while the rates of resistance against piperacillin/tazobactam sodium and cefoperazone/sulbactam was $11.1 \%$ and $44.4 \%$, respectively, with no resistance to imipenem, which can be used in clinical practice. As reported in the literature, plasmid-mediated ESBL production is often associated with other resistance genes, showing multidrug resistance, which was also supported by our results. ${ }^{19}$ The resistance rates of ESBL-producing strains against ciprofloxacin was $77.8 \%$, and that of non-ESBL-producing strains was $12.5 \%$, with a significant difference between the two groups.

Seven strains of ESBL-producing K. pneumoniae were detected in the current study. Five of these were collected from patients in the EICU from November 2012 to February 2013, with close time of occurrence, similar resistance profiles, and concentrated trend of the spatial distribution of the same clone strain, suggesting cross-contamination or an unknown common source of contamination existing in the EICU. To verify its homology, plasmid profiles were determined, showing a similar enzyme digestion profile in the five strains, indicating that bacteria with the same origin of clone caused this outbreak in the EICU. There are many reports of hospital infection caused by ESBL-producing bacteria, mostly occurring in the pediatric ward, ICU, and oncology ward. Taking the clinical conditions of the current study into consideration, risk factors that could lead to ESBL-producing bacterial infection include some of the factors that are described in the following. 1) Host factors: The nine patients infected by ESBL-producing bacteria were all old people with an average age of 67.9 years, who suffered degeneration of vital organ function and immunological defense ability complicated with multiple primary diseases (eg, chronic obstructive pulmonary disease, tumor, or diabetes). 2) Environmental factors: Tenover and Hughes ${ }^{20}$ emphasized that environmental conditions often promote drug resistance gene mutations resulting in the emergence of new phenotypes. 3) Interventional factors: During mechanical ventilation, tracheal intubation disrupts the normal barrier of the epiglottis, weakens the cough reflex and ciliary movement, and reduces the defensive function of the upper respiratory tract. These series of events made the nasopharyngeal bacteria cumulate around the catheter balloon and 
leak into the lung tissue with the retained secretion, causing pulmonary infection eventually. At the same time, the lung stores more than one-third of neutrophils outside the bone marrow and also secretes a variety of highly active cytokines, such as tumor necrosis factor $\alpha$ and interleukin-1 $\beta$, which facilitate the spread of inflammation. $\left.{ }^{21} 4\right)$ Antibiotic factors: All patients with ESBL-producing bacterial infection received third-generation cephalosporins before bacterial isolation, especially ceftazidime and cefotaxime, indicating that the use of broad-spectrum antibiotics is an important selection factor for ESBL-producing bacteria. 5) Pathogen factors: ESBL-producing bacteria may be carried by plasmids, which can be transferred and diffused among different strains through conjunction, transformation, and transduction, resulting in widespread antibiotic resistance. ${ }^{22}$ In view of the high risk factors of infection caused by ESBL-producing bacteria, the rational use of antibiotics is an important means to fight multidrug-resistant bacteria. ESBL-producing bacteria usually colonize the gastrointestinal tract; therefore, selective decontamination of the digestive tract could be a control measure in these patients. ${ }^{23}$ Furthermore, improving disinfection and isolation measures, strict aseptic procedures, and barrier nursing are also important methods of reducing hospital infection.

Antibiotics selected for the initial empirical treatment of VAP should cover all possible pathogens, including A. baumannii, P. aeruginosa, P. maltophilia, and MRSA, etc, to reduce the mortality of VAP. ${ }^{6}$ Most of the patients in this study were empirically treated with combined use of antibiotics. However, some patients failed because the antibiotic spectrum did not cover Staphylococcus, or the broad-spectrum antibiotics were ineffective against highresistant Gram-negative bacteria. Even if the antibiotics were adjusted according to the results of drug susceptibility, the prognosis of some patients remained unchanged. Therefore, monitoring bacterial resistance in VAP patients, timely understanding of bacterial distribution and drug resistance, and establishing the best empirical therapy strategy in this region is necessary in order to slow down the selective pressure of antibiotic resistance to bacteria, delay the emergence of bacterial resistance, and reduce the mortality of VAP. In view of the epidemic trend of multidrug-resistant bacterial infection, strengthening the management of ward environment, isolating patients with ESBL infections from those with no ESBLs infection, standardizing medical and nursing practices, and paying attention to the control of hospital infection links can block the occurrence and spread of drug-resistant bacteria.

\section{Conclusion}

Gram-negative bacteria were still the most important pathogens in VAP and had high resistance to antibiotics. ESBL-producing strains are mainly expressed by Enterobacteriaceae. Plasmids played an important role in the spread of antibiotic resistance among bacteria.

\section{Acknowledgments}

We thank all the authors and people who helped us to carry out this work. This work was funded in part by Liaoning Science and Technology Project of China (Grant No. 2015020527).

\section{Disclosure}

The author reports no conflicts of interest in this work.

\section{References}

1. Shaikh S, Fatima J, Shakil S, Rizvi SM, Kamal MA. Antibiotic resistance and extended spectrum beta-lactamases: Types, epidemiology and treatment. Saudi J Biol Sci. 2015;22(1):90-101.

2. Rao SP, Rama PS, Gurushanthappa V, Manipura R, Srinivasan K. Extended-Spectrum Beta-Lactamases Producing Escherichia coli and Klebsiella pneumoniae: a Multi-Centric Study Across Karnataka. J Lab Physicians. 2014;6(1):7-13.

3. Uz Zaman T, Aldrees M, Al Johani SM, Alrodayyan M, Aldughashem FA, Balkhy HH. Multi-drug carbapenem-resistant Klebsiella pneumoniae infection carrying the OXA-48 gene and showing variations in outer membrane protein 36 causing an outbreak in a tertiary care hospital in Riyadh, Saudi Arabia. Int J Infect Dis. 2014;28:186-192.

4. Flores-Carrero A, Labrador I, Paniz-Mondolfi A, Peaper DR, Towle D, Araque M. Nosocomial outbreak of extended-spectrum $\beta$-lactamaseproducing Enterobacter ludwigii co-harbouring CTX-M-8, SHV-12 and TEM-15 in a neonatal intensive care unit in Venezuela. J Glob Antimicrob Resist. 2016;7:114-118.

5. Helldal L, Karami N, Welinder-Olsson C, Moore ER, Åhren C. Evaluation of MLVA for epidemiological typing and outbreak detection of ESBL-producing Escherichia coli in Sweden. BMC Microbiol. 2017;17(1):8.

6. Vincent JL. Ventilator-associated pneumonia: a review. J Hosp Infect. 2004;57(4):272-280.

7. Rhodes NJ, Cruce CE, O'Donnell JN, Wunderink RG, Hauser AR. Resistance Trends and Treatment Options in Gram-Negative VentilatorAssociated Pneumonia. Curr Infect Dis Rep. 2018;20(2):3.

8. Xie DS, Xiong W, Lai RP, et al. Ventilator-associated pneumonia in intensive care units in Hubei Province, China: a multicentre prospective cohort survey. J Hosp Infect. 2011;78(4):284-288.

9. Restrepo MI, Anzueto A, Arroliga AC, et al. Economic burden of ventilator-associated pneumonia based on total resource utilization. Infect Control Hosp Epidemiol. 2010;31(5):509-515.

10. Vandecandelaere I, Coenye T. Microbial composition and antibiotic resistance of biofilms recovered from endotracheal tubes of mechanically ventilated patients. Adv Exp Med Biol. 2015;830:137-155.

11. Chittawatanarat K, Jaipakdee W, Chotirosniramit N, Chandacham K, Jirapongcharoenlap T. Microbiology, resistance patterns, and risk factors of mortality in ventilator-associated bacterial pneumonia in a Northern Thai tertiary-care university based general surgical intensive care unit. Infect Drug Resist. 2014;7:203;16:203-210.

12. Abdalhamid B, Hassan H, Itbaileh A, Shorman M. Characterization of carbapenem-resistant Acinetobacter baumannii clinical isolates in a tertiary care hospital in Saudi Arabia. New Microbiol. 2014;37(1):65-73. 
13. Li H, Liu F, Zhang Y, et al. Evolution of carbapenem-resistant Acinetobacter baumannii revealed through whole-genome sequencing and comparative genomic analysis. Antimicrob Agents Chemother. 2015;59(2):1168-1176.

14. Wieczorek P, Sacha P, Czaban S, et al. Distribution of AdeABC efflux system genes in genotypically diverse strains of clinical Acinetobacter baumannii. Diagn Microbiol Infect Dis. 2013;77(2):106-109.

15. Abdallah M, Benoliel C, Ferreira-Theret P, Drider D, Dhulster P, Chihib NE. Effect of culture conditions on the resistance of Pseudomonas aeruginosa biofilms to disinfecting agents. Biofouling. 2015;31(1):49-59.

16. Laverty G, Gorman SP, Gilmore BF. Biomolecular Mechanisms of Pseudomonas aeruginosa and Escherichia coli Biofilm Formation. Pathogens. 2014;3(3):596-632.

17. Wu N, Chen BY, Tian SF, Chu YZ. The inoculum effect of antibiotics against CTX-M-extended-spectrum $\beta$-lactamase-producing Escherichia coli. Ann Clin Microbiol Antimicrob. 2014;13:45

18. Horner C, Fawley W, Morris K, Parnell P, Denton M, Wilcox M. Escherichia coli bacteraemia: 2 years of prospective regional surveillance (2010-12). J Antimicrob Chemother. 2014;69(1):91-100.
19. Shaikh S, Fatima J, Shakil S, Rizvi SM, Kamal MA. Antibiotic resistance and extended spectrum beta-lactamases: Types, epidemiology and treatment. Saudi J Biol Sci. 2015;22(1):90-101.

20. Tenover FC, Hughes JM. The challenges of emerging infectious diseases. Development and spread of multiply-resistant bacterial pathogens. JAMA. 1996;275(4):300-304.

21. Nickles HT, Sumkauskaite M, Wang X, Wegner I, Puderbach M, Kuebler WM. Mechanical ventilation causes airway distension with proinflammatory sequelae in mice. Am J Physiol Lung Cell Mol Physiol. 2014;307(1):L27-L37.

22. Dhara L, Tripathi A. Genetic and structural insights into plasmidmediated extended-spectrum $\beta$-lactamase activity of CTX-M and SHV variants among pathogenic Enterobacteriaceae infecting Indian patients. Int J Antimicrob Agents. 2014;43(6):518-526.

23. Schnabel RM, Scholte JB, Van Der Velden KE, Roekaerts PM, Bergmans DC. Ventilator-associated pneumonia rates after introducing selective digestive tract decontamination. Infect Dis (Lond). 2015;47(9) $50-653$.
Infection and Drug Resistance

\section{Publish your work in this journal}

Infection and Drug Resistance is an international, peer-reviewed openaccess journal that focuses on the optimal treatment of infection (bacterial, fungal and viral) and the development and institution of preventive strategies to minimize the development and spread of resistance. The journal is specifically concerned with the epidemiology of antibiotic

\section{Dovepress}

resistance and the mechanisms of resistance development and diffusion in both hospitals and the community. The manuscript management system is completely online and includes a very quick and fair peerreview system, which is all easy to use. Visit http://www.dovepress.com/ testimonials.php to read real quotes from published authors. 